\title{
High Presence Communication between the Earth and International Space Station
}

\author{
Tetsuro Ogi, Yoshisuke Tateyama, and Yosuke Kubota \\ Graduate School of System Design and Management, Keio University \\ 4-1-1 Hiyoshi, Kohoku-ku, Yokohama 233-8526, Japan \\ ogi@sdm.keio.ac.jp, \\ tateyama@sdm.keio.ac.jp, yosuke-k21@z2.keio.jp
}

\begin{abstract}
In this study, in order to realize high presence communication with the astronaut who is staying on the ISS, the experiment on remote communication using the technologies of 2D/3D conversion, immersive dome display, and sharing space among multiple sites were conducted. In this case, biological information such as electrocardiogram, thermal image, and eye movement were measured to evaluate the sense of presence, and the tendency that the user felt the high presence sensation when experiencing the high resolution three-dimensional stereo image. From these results, we can understand that high presence communication between the earth and the ISS was realized.
\end{abstract}

Keywords: Tele-immersion, High Presence Sensation, Biological Information, 2D/3D Conversion, International Space Station.

\section{Introduction}

Recently, outer space has become not only the space that is flown through by the rocket or space shuttle but also habitation space where human can stay for a long time, according to the development of ISS (International Space Station) [1]. Therefore, it is one of the important demands to realize high presence communication between the human who is on the earth and the astronaut who is staying on the space station. In order to solve such a demand, the researches about the high presence communication using the virtual reality or tele-immersion technologies are expected to be an effective approach.

In November, 2012, the authors got an opportunity to perform a communication event with the ISS, and we conducted an experiment on high presence communication between the earth and the ISS in this event. In the communication event, seven sites were connected to the ISS simultaneously, and the graduate students and the elementary and junior high school students asked questions from Keio University to astronaut Akihiko Hoshide staying on the ISS. In this event, in order to realize high presence communication, we applied several technologies such as high resolution 3D image, immersive dome display, and sharing space among multiple sites. This paper describes the experiment about the high presence communication between the earth and the ISS that was conducted in this event. 


\section{High Presence Communication}

In the communication event with the ISS, the image sent from the ISS was received at several sites simultaneously and it was shared among all sites while performing each event respectively.

As a conversation place where the students talk to the astronaut on the ISS, CDF (Concurrent Design Facility) room in Keio University was used [2], where the symposium entitled "Information Technology and Space Age" was performed. In addition, extension lecture about space engineering was held in the main hall in the same building as the CDF room, and the events for children were held at citizen halls in Setagaya-ku and Minamisanriku-cho. The video image transmitted from the ISS was once received in the CDF room, and it was distributed to other sites. Moreover, the air dome display that was built temporarily in Keio University, and the conference rooms at the University of Tokyo and Kyoto University were also connected to the $\mathrm{CDF}$ room through the network, and the video image edited in the CDF room was distributed to each site.

In this study, the experiment was conducted for the purpose of realizing high presence communication between the earth and the ISS. Although various systems that use stereo image, high resolution image, or large screen image have been developed to represent or transmit high presence sensation, the equipment that can be used in the current ISS is restricted. Therefore, in this experiment, we gave up realizing high presence mutual communication and aimed at generating high presence sensation only at the earth side.

As the concrete methods about the high presence communication, the following technologies were applied;

\section{2D/3D conversion}

2. Immersive dome display

3. Sharing space among multiple sites.

\section{Network System}

Figure 1 shows the construction of the network system that was built in this experiment. Though the network for the image transmission from the ISS to NASA and JAXA (Japan Aerospace Exploration Agency) is always secured and monitored, the network to other places must be constructed by ourselves. In this experiment, the network environment in which the communication image that is transmitted from the ISS to JAXA Tsukuba Space Center can be sent to Keio University through SINET (Science Information Network) or Tsukuba WAN was constructed. Since the communication load in SINET changes depending on the period of time, another path of the network using Tsukuba WAN and JGN-X (Japan Gigabit Network eXtreme) was prepared for backup. These networks were connected to the CDF room in Keio University through WIDE (Widely Integrated Distributed Environment) network. 


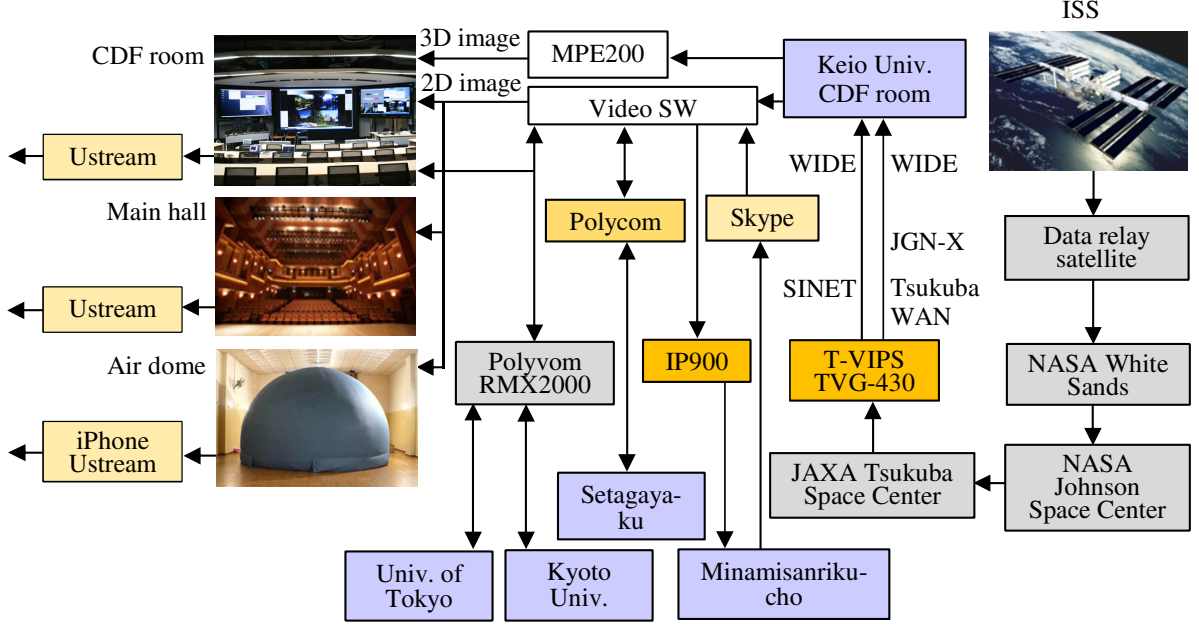

Fig. 1. Network construction of the remote communication between the earth and ISS

As for the method of the image transmission, IP transmission equipment, video conferencing system, IP TV phone, and video streaming service were used for different purposes. Since the image that was transmitted from JAXA Tsukuba Space Center to the CDF room at Keio University was used for the conversation between the earth and the ISS directly as well as for the shared image among all sites, T-VIPS TVG-430 of the IP transmission equipment based on JPEG2000 format was used in consideration of the image quality and real-time processing.

The video image received at the CDF room was then distributed to other sites. Since the main hall and the air dome were placed in the same building as the CDF room, the video image was transmitted from the CDF to two sites using the coaxial cable via a distributor. In addition, the video images were distributed mutually among three sites of the CDF room, the University of Tokyo, and Kyoto University using a multi-point HD video conference connection server Polycom RMX2000. At the citizen hall in Setagaya-ku, HD video conferencing system Polycom HDX8005 was used independently, in order to exchange the images mutually between the CDF room and the citizen hall and to use the image of the other site in each event. Though the image of the ISS was transmitted from the CDF room to the citizen hall in Minamisanriku-cho using the H.264 format by the IP transmission equipment Fujitsu IP900, the image captured at Minamisanriku-cho was sent to the CDF room using Skype of IP TV phone to transmit only the atmosphere of the hall.

Moreover, the video images of the events held in the CDF room and main hall at Keio University were broadcasted on the Internet using Ustream video streaming service. The video image captured at the air dome in Keio University was broadcasted using the iPhone Ustream, and this image was also used for the communication between the dome display and the other sites. Namely, in this system, the images were transmitted using the appropriate methods in consideration of the network bandwidth, time delay, the usability of the communication equipment, and so on. 


\section{Communication Using 3D Image}

First, in this study, in order to realize high presence communication, high resolution 3D image was used in the CDF room. Since a stereo camera cannot be installed in the ISS, 2D/3D conversion technology was applied to the transmitted monocular HD image in real time and the three-dimensional stereo image was generated. The communication time given by JAXA was about 20 minutes, and data relay satellite was switched in the middle of the communication. Therefore, the transmitted twodimensional video image was displayed without conversion for 10 minutes of the first half, and the converted three-dimensional video image was displayed for 10 minutes of the second half. The image processor SONY MPE-200 was used for 2D/3D conversion, and the generated three-dimensional stereo image was projected onto the 180 -inch screen using the stacked $4 \mathrm{~K}$ projectors of SONY SRX-S110 through the polarizing filter.

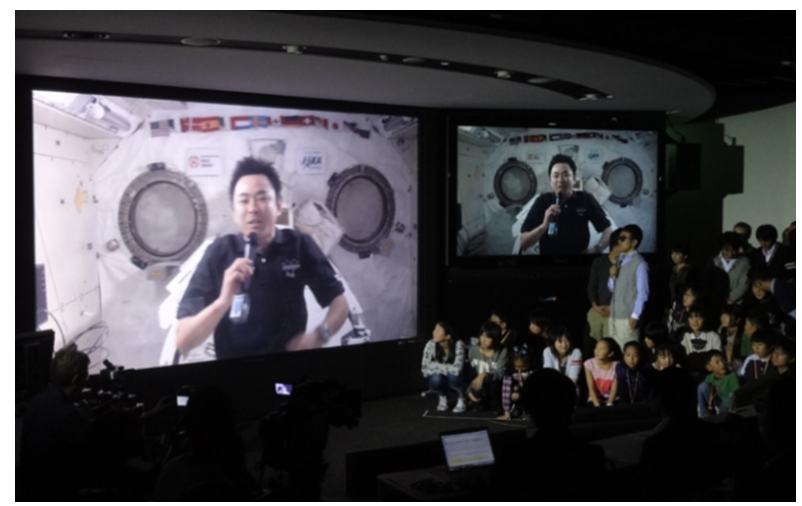

CJAXA

Fig. 2. Conversation between student and astronaut in ISS using 3D image

In general, in the method of $2 \mathrm{D} / 3 \mathrm{D}$ conversion, three-dimensional scene is constructed by recognizing objects in the monocular image and adding the information of binocular parallax to each object. In this case, the added information of the binocular parallax is not necessarily accurate, because it is generated automatically according to the conversion algorithms such as the focal point analysis. In this experiment, since the full-scale model of Japanese experiment module "Kibo" in the ISS exists in JAXA Tsukuba Space Center, it was possible to measure the size of the model and to adjust the value of the binocular parallax so that the image can be represented as real scale. In this case, the parameters for binocular parallax were adjusted using the video image that was recorded in the previous event, so that the image of the inner space of "Kibo" was represented as three-dimensional real scale 
image. However, the image of astronaut Hoshide that was generated in the real-time communication was represented as larger size, because the positions of the astronaut and the video camera in the actual communication were unknown. Figure 2 shows the three-dimensional stereo image of astronaut Hoshide that was projected onto the 180inch screen. In the CDF room, the graduate students and the elementary and junior high school students talked with astronaut Hoshide while looking at the threedimensional stereo image.

The purpose of this experiment includes the evaluation of the high presence sensation that was represented in the remote communication as well as the realization of the real-time communication between the earth and the ISS. However, the questions of "What is the high presence sensation?" and "How the high presence sensation can be measured?" are not clearly defined. In order to solve this problem, we have examined the method of evaluating the high presence sensation based on the measurement of biological information. From the past research, we have found that when the persons are experiencing high presence images, the value of RRV (variance of R-R intervals) measured by electrocardiograph becomes lower [3], the nose temperature measured by thermal camera does not fall [4], and the view point measured by eye tracker moves frequently. This means that when the person is experiencing the high presence sensation, he is concentrating his attention, feeling few mental stresses, and interested in a lot of objects.

In this experiment, we measured the electrocardiogram, the thermal image, and the eye movement of the subject who was sitting on the center seat of the front row in order to evaluate the presence felt by the subject, and compared the sensation when he was seeing the two-dimensional image and three-dimensional image of the astronaut.

Figure 3 shows the change in the value of RRV. Though the average of RRV was $0.715\left(10^{-3} \mathrm{sec}^{2}\right)$ when seeing the two-dimensional image, it was $0.657\left(10^{-3} \mathrm{sec}^{2}\right)$ when seeing the three-dimensional image, and there was significant difference between them. Figure 4 shows the change in the nose temperature and forehead temperature. The average of the difference between nose temperature and forehead temperature was 0.276 (degrees) and 0.141 (degrees) when seeing the two-dimensional image and three-dimensional image, respectively. And there was significant difference between them. As for the eye movement, the frequency of view point movement was 1.064 (times per second) and 1.147 (times per second) when seeing the two-dimensional image and three-dimensional image, respectively. And there was a tendency that the view point moves a little frequently when seeing the three-dimensional image. From these results, we can recognize that when the subject was experiencing the threedimensional image of astronaut Hoshide, the value of RRV became low, the nose temperature did not fall, and view point moved frequently. Namely we can understand that the subject experienced the high presence communication with the astronaut Hoshide by using the high resolution three-dimensional stereo image. 


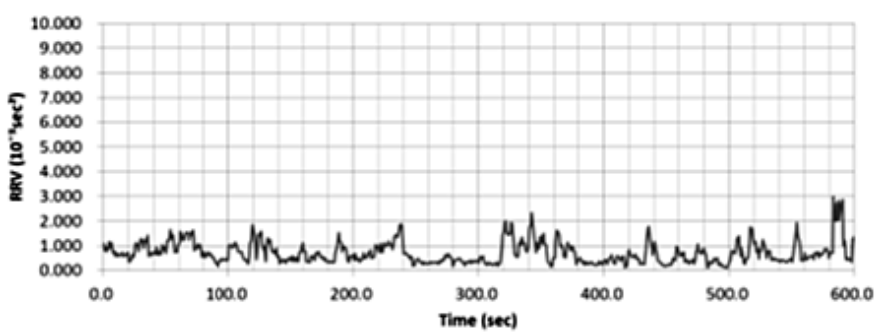

(a) 2D image

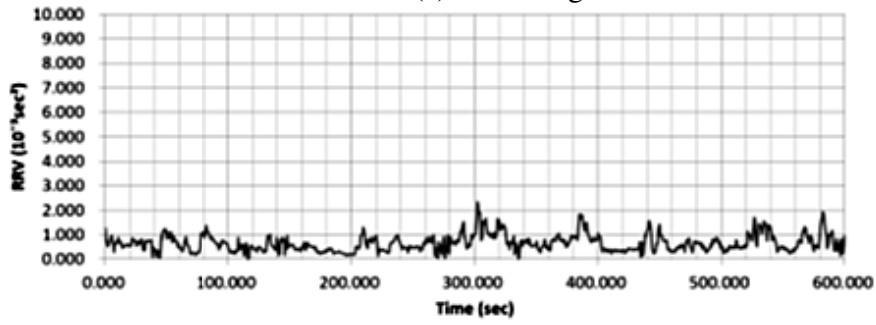

(b) 3D image

Fig. 3. RRV value when seeing (a) $2 \mathrm{D}$ image and (b) 3D image

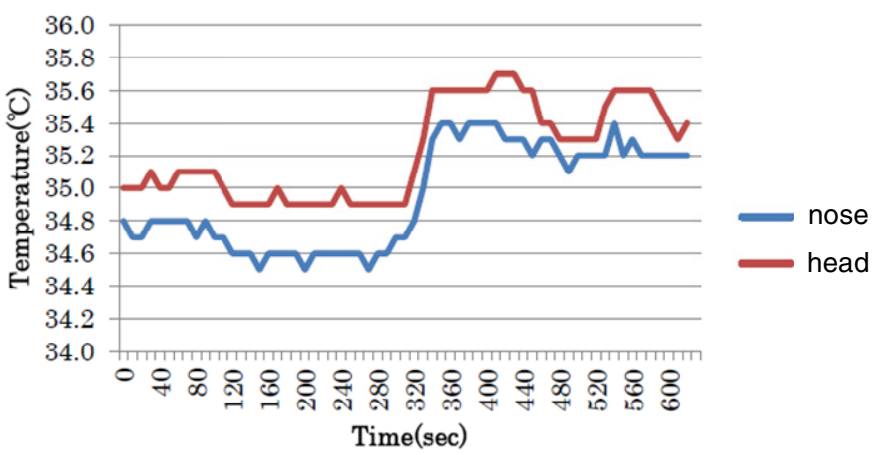

(a) 2D image

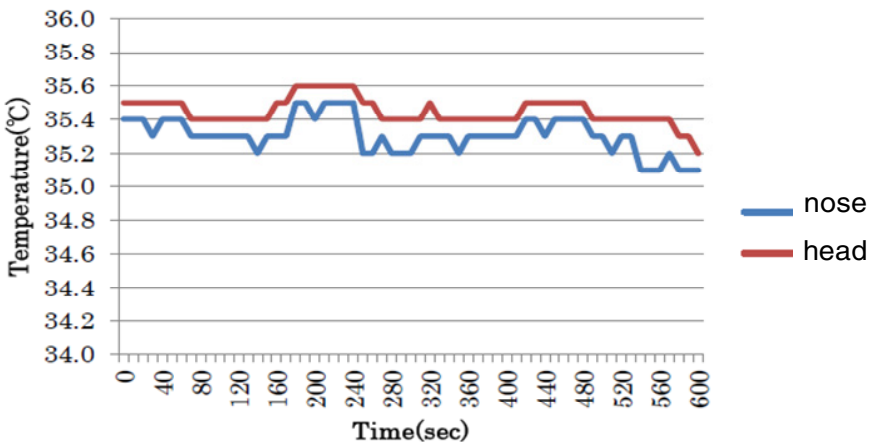

(b) 3D image

Fig. 4. Nose and head temperature when seeing (a) 2D image and (b) 3D image 
Moreover, as subjective evaluation, we conducted a questionnaire survey to the participants in the communication event performed in the CDF room. In the questionnaire, the questions about the communication using the two-dimensional image and the three-dimensional image shown in Table 1 were asked. Participants were asked to answer each question using five-grade system such as "agree $(+2)$, somewhat agree $(+1)$, neither agree nor disagree $(0)$, somewhat disagree $(-1)$, disagree $(-2) "$. In the table, the average values and standard deviations of 38 persons who answered the questionnaire and the results of t-test for each question are shown. From the result, we can see that there is significant difference in the questions of "I felt that astronaut was talking to me" and "I felt that I was in the same space with astronaut". Therefore, we can understand that the high presence sensation was represented by using the high resolution three-dimensional image from the subjective evaluation.

Table 1. Result of questionnaire about $2 \mathrm{D}$ and $3 \mathrm{D}$ image

\begin{tabular}{l|ccc}
\hline \multicolumn{1}{c|}{ question } & 3D image & 2D image & t-test \\
\hline I felt three-dimensional sensation. & 1.16 & -0.34 & $\mathrm{P}=0.000$ \\
I concentrated attention. & 1.21 & 0.71 & $\mathrm{P}=0.026$ \\
I felt excited. & 0.89 & 0.21 & $\mathrm{P}=0.047$ \\
I felt tired. & -0.34 & -0.92 & $\mathrm{P}=0.029$ \\
I felt that astronaut was talking to me. & 0.63 & -0.08 & $\mathrm{P}=0.007$ \\
I felt that I was in the same space with astronaut. & 0.58 & -0.13 & $\mathrm{P}=0.006$ \\
\hline
\end{tabular}

$(-2)$ disagree -- $(0)$-- agree $(+2)$

\section{$5 \quad$ Immersive Dome Display}

Next, the immersive dome display was used to represent the high presence sensation. In the dome environment, it is known that the user can experience immersive sensation from the frameless image with wide field of view. In addition, it is also known that the user can feel three-dimensional sensation from the monocular image with wide field of view without wearing 3D glasses [5]. In particular, in the content of the remote communication with the ISS, we can expect the effect that the planetarium-like environment of the dome display reinforces the sense of presence by making the user imagine the space.

In this experiment, the air dome system of CUBEX Dome with 5 meters in diameter was built temporarily in the same building as the CDF room. In this system, the image was projected onto the dome screen by using one projector to which 180 degrees fish-eye lens was attached. Though, in general, the image projected by the fish-eye projector contains large distortion, in this system, the transmitted image was directly projected onto the screen without correcting the distortion, because the distortion based on the fish-eye lens was reduced by the shape of the dome screen.

Figure 5 shows the appearance of the air dome system that was installed in this experiment and figure 6 shows the image of astronaut Hoshide that was projected onto the dome screen. Though the number of users who can experience the air dome simultaneously was about ten, the comments such as "The immersion felt in the dome display was suitable for the representation of the space." and "The size of the 
dome display was suitable to represent the image of the astronaut with high presence sensation." were gotten from the users. From these comments, we can see that the user experienced the high presence image effectively in the dome environment. Although we planned to use the large-scale planetarium at the beginning, it is thought that this air dome was a suitable size to represent the internal scene of the ISS.

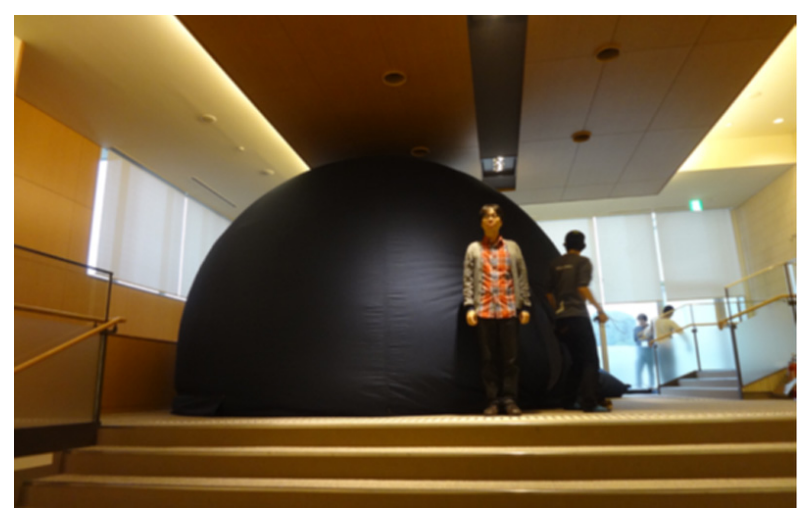

Fig. 5. Air dome with 5 meters in diameter built in the building

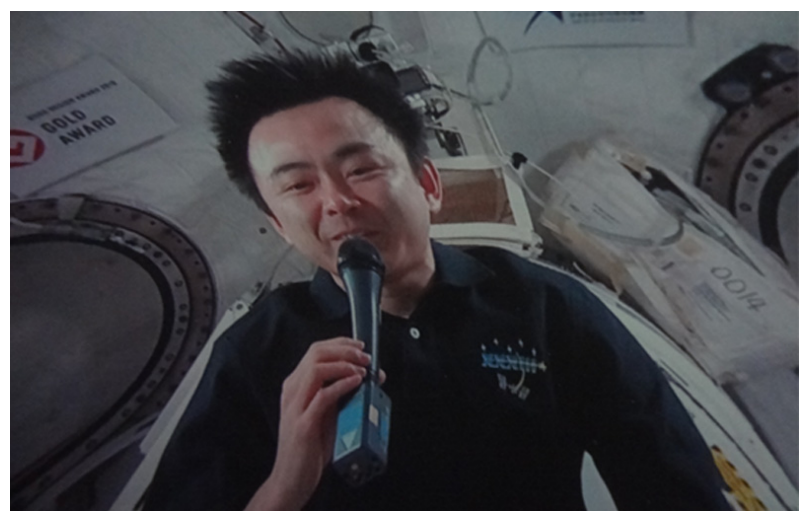

(C) JAXA

Fig. 6. Image of astronaut Hoshide projected onto the dome screen

\section{$6 \quad$ Sharing Space among Multiple Sites}

This communication event was performed by connecting seven sites, such as the CDF room, the main hall, and the air dome in Keio University, the citizen halls in Setagaya-ku and Minamisanriku-cho, and the conference rooms in the University of Tokyo and Kyoto University through the network. Though the several events of the symposium, the extension lecture, and the quiz were performed in the CDF room, 
main hall at Keio University, and the citizen halls in Setagaya-ku and Minamisanrikucho respectively, whole event was also constructed in all sites by sharing information based on the image transmission. In particular, the sensation of sharing space was generated by displaying the same image at all sites while performing the communication between the earth and the ISS.

We can say that the participants experienced a kind of parallel reality that consisted of several sites including the ISS. Parallel reality is a concept in which the user can experience several real worlds simultaneously by using various kinds of image transmission technologies. In this experiment, space sharing among multiple sites was realized by using various image transmission technologies such as IP transmission equipment, video conferencing system, IP TV phone, and video streaming service.

Particularly, since the CDF room that was used for the conversation with the ISS consists of 180-inch 4K3D screen and two 108-inch LCD monitors placed at both sides, three-dimensional stereo image and other two kinds of images can be displayed at the same time. Moreover, since the $4 \mathrm{~K}$ screen can display four divided high definition images, it can be used as multi-display environment where a total of six kinds of images can be displayed. Figure 7 shows that the users were experiencing the events of multiple sites simultaneously by looking at the images that were sent from other sites.

In this experiment, the participants in each hall were able to experience the events in other halls simultaneously by connecting each site mutually based on the image transmission, and the sensation of sharing space among multiple sites was generated. Namely, we can understand that this experimental event generated the consciousness of being experiencing one of the real worlds that includes the space environment for many participants.

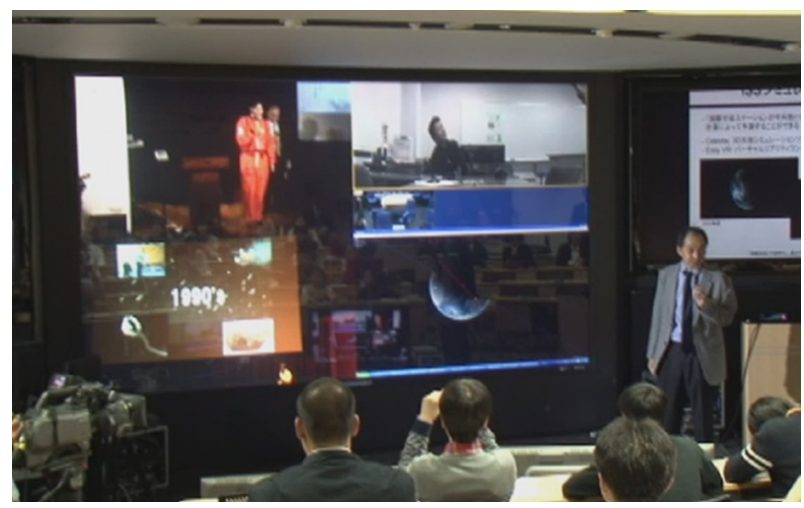

Fig. 7. Experience of sharing space using multi-display environment

\section{Conclusions}

In this study, the experiment on high presence communication between the earth and the ISS was conducted. In the experiment, the technologies of $2 \mathrm{D} / 3 \mathrm{D}$ conversion, immersive dome display, and sharing space among multiple sites were applied to 
realize the high presence communication. In particular, in the experiment of $2 \mathrm{D} / 3 \mathrm{D}$ conversion, the sensation of the presence felt by the user was verified based on the measurement of the biological information.

Although each technology of the image transmission used in this experiment was completed technology, it was not easy to use them simultaneously and manage the whole system, and some small troubles such as the interruption of the sound happened in the communication event. Future work will include acquiring more experiences of the communication experiment and establishing the technology that can be used more easily even in the space station.

Acknowledgements. This research was supported by Keio Gijuku Academic Development Funds and G-COE (Center of Education and Research of Symbiotic, Safe and Secure System Design) program at Keio University. We thank Prof. Naohiko Kohtake, Prof. Naohisa Ohta, and Prof. Akira Kato of Keio University, Mr. Yutaka Kaneko of JAXA, Mr. Tsuyoshi Doi of Yomiuri Shinbun, and other event staff members for their support.

\section{References}

1. Okami, Y.: Space Station and Supporting Engineering. Journal of the Institute of Electrical Engineers of Japan 121(3), 199-202 (2001)

2. Ogi, T., Tsubouchi, D.: Development of Concurrent Design Environment Using Super High Definition Image. In: 2010 Asian Conference on Design \& Digital Engineering (ACDDE 2010), pp. 85-88 (2010)

3. Hirose, M., Nakagaki, Y., Ishii, T.: Application for Operational Management using R-R Intervals of ECG Measured by Optical Sensor. Proc. of the Japan Society of Mechanical Engineers 884(5), 82-84 (1984)

4. Toma, T., Ogi, T.: Influence of Live Classic Concert on Stress and Relaxation in 3D High Presence Environment. In: Asian Conference on Design and Digital Engineering (ACDDE 2011 Proceeding II), pp. 503-506 (2011)

5. Ogi, T., Tateyama, Y., Lee, H., Furuyama, D., Seno, T., Kayahara, T.: Creation of Three Dimensional Dome Contents Using Layered Images. In: The 1st International Symposium on Virtual Reality Innovations (IEEE ISVRI), pp. 253-258 (2011) 